\title{
Roles of surgery in complicated necrotizing pneumonia
}

\section{Editorial}

Necrotizing pneumonia (NP), an uncommonly severe complication of benign-course community-acquired pneumonia (CAP) with increasing incidence that is generally related to poor clinical outcomes, particularly in adults, but uncommon in childhood population. CAP can be complicated by lung abscess, pleural empyema (empyema thoracis), necrotizing pneumonia, lung gangrene, and variable number of pneumatoceles or thin-wall cavities based on the extent of lung parenchymal involvement, severity or degree of sepsis, roentgenographic characteristics, time course, and degree or area of inflammation and necrosis. On computed tomography (CT), NP is defined as patchy infiltration with microabscesses and decreased lung perfusion. The involved mechanism is thrombotic occlusion of the alveolar capillaries related to adjacent inflammation, contributing to ischemia, and finally necrosis of the lung parenchyma. The classification of NP is divided into:

a) Complicated NP (with large cavities involving $>50 \%$ of the involved lobe or with massive lung necrosis)

b) Uncomplicated NP (with less severe CT features).

On roentgenographic findings, NP is presented by pulmonary inflammation with multiple small lung cavities, lung consolidation, and lung necrosis. NP is frequently accompanied by some degree of vascular compromise leading to medical treatment failure due to inadequate antimicrobial administration that there are no clear management guidelines for such complicated NP patients and limited literature involving surgical management.

Recent case reports demonstrated that the pathogens isolated in lung tissue and pleural fluid were Streptococcus pneumoniae, Staphylococcus aureus, Burkholderia pseudomallei, Mycobacterium tuberculosis, and Acinetobacter baumanii. Other recent case series revealed that the most reported adult cases were frequently related to Staphylococcus aureus, whereas the most common identified pathogenic organisms in pediatric patients was Streptococcus pneumoniae, followed by Staphylococcus aureus and Klebsiella pneumoniae. Although no clear guidelines exist for patients with complicated NP, surgery is sometimes offered upon failure of
Volume 7 Issue I - 2019

\author{
Attapon Cheepsattayakorn,' Ruangrong \\ Cheepsattayakorn ${ }^{2}$ \\ ' $10^{\text {th }}$ Zonal Tuberculosis and Chest Disease Center, Chiang Mai, \\ Thailand \\ ${ }^{2}$ Department of Pathology, Faculty of Medicine, Chiang Mai \\ University, Thailand
}

Correspondence: Attapon Cheepsattayakorn, I0th Zonal Tuberculosis and Chest Disease Center, 143 Sridornchai Road Changklan Muang Chiang Mai, 50100, Thailand, Tel 6653 140767, 6653 276364, Fax 6653 I40773, Email Attapon1958@gmail.com

Received: February 28, 2019 | Published: February 28, 2019 adequate medical treatment, frequently as a life saving therapy. The indications for surgery include persistent fever despite aggressive medical treatment (antimicrobials, thoracocentesis, chest intubation or pigtail catherization, and fibrotic therapy), major hemoptysis, lung abscess, pleural empyema, lung gangrene, sepsis, and persistent respiratory distress. The surgical operations include thoracoscopic decortications without lung resection, sublobar resection, wedge resection, lobectomy, and bilobectomy, whereas preoperative imaging studies, particularly CT are critical in managing NP.

In conclusion, complicated pediatric NP has more preoperative morbidity and major postoperative complications and requires longer postoperative stay, compared to uncomplicated NP. Cases with massive pulmonary necrosis, preoperative intubation for pneumothorax, respiratory distress, or hemolytic uremic syndrome may require formal lobectomy. Surgical procedures for NP must be tailor-made for different patient populations with varying degree of lung parenchymal and pleural involvement.

\section{Acknowledgments}

None.

\section{Conflicts of interest}

Author declares that there are no conflicts of interest. 